\title{
112
}

\section{VALUTAZIONE DI UN NUOVO TEST IMMUNOENZIMATICO PER IL DOSAGGIO} AG-AB DELL'HCV

\author{
Alecci A., Fontanucci M.,Venturi D. \\ Settore Sierologia A.C. Laboratorio Analisi Cliniche \\ Ospedale S.M. Annunziata ASL 10 Firenze
}

Il kit Monolisa HCV Ag-Ab Ultra della ditta Bio-Rad è il primo dosaggio immunoenzimatico che permette di evidenziare contemporaneamente antigene e anticorpo associati all'infezione da HCV . Abbiamo valutato specificità, sensibilità e impatto di questo test confrontandolo con i due diversi tests di screening usati nel nostro Laboratorio di Sierologia. Tutti i campioni borderline al test anti HCV Architect metodica CMIA automatizzata vengono saggiati anche con il test immunoenzimatico Ortho HCV 3.0 Elisa con metodica manuale.

200 campioni di siero della routine giornaliera risultati negativi per gli anticorpi anti HCV con il test anti HCV Architect sono stati saggiati con il kit Monolisa HCV Ag-Ab Bio-Rad; si è evidenziata la positivita' di un solo campione. 123 sieri, 
selezionati nel periodo maggio-giugno 2005, costituiti da campioni risultati borderline con il test anti HCV Architect sono stati saggiati con il test Ortho HCV 3.0 Elisa ed il test Monolisa Ag-Ab Bio-Rad .

Abbiamo evidenziato quanto segue: dei 44 campioni borderline, ma inferiori al cut off $(1.0 \mathrm{~S} / \mathrm{CO}$ del kit anti $\mathrm{HCV}$ Architect ) abbiamo avuto 39 negativi e 5 positivi con il test Ortho HCV 3.0 e 38 negativi e 6 positivi con il test Monolisa $\mathrm{Ag}-\mathrm{Ab}$.

Dei 79 campioni borderline ma superiori al cut off (1.0 $\mathrm{S} / \mathrm{CO}$ ) abbiamo avuto 42 negativi e 37 positivi con il test Ortho HCV 3.0 e 39 negativi e 40 positivi con il test Monolisa Ag-Ab.

Questi dati preliminari evidenziano una buona correlazione fra il test della ditta Ortho, che ha sempre avuto un'ottima specificità per i campioni borderline, ed il nuovo test della ditta Bio-Rad,.

In questo saggio inoltre è stato ridimensionato il numero dei campioni borderline e si è anche evidenziata la presenza al test di screening di 3 positività in più che altrimenti avremmo perduto. 